\title{
The development of geoinformation system elements for solving application tasks in an AutoCad Map 3D environment*
}

\author{
Larisa A. Ginis \\ Southern Federal University \\ Taganrog, Russia \\ e-mail: gla@sfedu.ru
}

\author{
Larisa V. Gordienko \\ Southern Federal University \\ Taganrog, Russia \\ lgordienko@sfedu.ru
}

\begin{abstract}
The purpose of this work is the description of experience of development separate subsystems of geographic information systems for implementation to different spheres of economy. Examples of implementation are the modules of geographic information systems developed by authors for different application areas: accounting of subjects of the land registry, road monitoring, calculation of insurance payments on vehicles. The basic structural elements of these geographic information systems are given, stages and a technique of their design are described.
\end{abstract}

Keywords-geoinformation system, programming, AutoCad Map 3D, AutoLisp, database, user interface, menu

\section{INTRODUCTION}

The geoinformation system (GIS) is an efficient means for processing spatial information. At the same time the main share of information has a spatial reference. It causes variety of applications of GIS: infrastructure of city, inventory, transport, dispatching and monitoring of critical objects, ecology, etc. Such practical demand resulted in a wide variety of software products based on GIS-technologies: from expensive fullfunction packets to GIS oriented to solution of specific application tasks. Table software complexes of ESRI, ERDAS, MapInfo in addition to high price require powerful system resources. Often the companies need GIS to solve the narrow specialized tasks. In this case, universal CAD, such as Autocad, can effectively be used to create the GIS.

\section{TASK SET}

The variety of applications of GIS provides differences in the principles of their development. These include methods of obtaining and storing spatial and attribute information, features of visualization of data, methods of analysis of map information.

In the development of geoinformation systems, it is necessary to consider system resources on the basis of which the functioning of the GIS is planned, the functional (set of functions of the system ) and temporal constraints. The main objective when constructioning GIS consists in obtaining the qualitative decision in case of the given set of restrictions:

$$
\left\{\begin{array}{l}
R(S) \cap R(F) \cap R(I) \leq R^{*} \\
G(W) \rightarrow \max
\end{array}\right.
$$

where $R(S)$ - expenses of system resources, $R(F)$ - the functional restrictions, $R(T)$ - time expenditure, $R^{*}$ is the vector of resource restrictions, $G(W)$ - function of the information value of the of the received decision, $W$ - working area of the decision.

In this work, the design features of the GIS are analyzed based on the principle of de-composition of the structural elements. The implementation of the stated task is confirmed by specific examples : insurance evaluation of vehicles on the basis of GIS-technologies, equipment of urban road network, accounting of objects of the land registry.

\section{Decision Methodology}

In the generalized case, the GIS model can be represented as follows [1]:

$$
<D, K, I, F>
$$

where $D$ - an attribute data store, $K$ - a map basis, $I-$ a user interface, $F-$ a function set.

The data store makes it possible to add the additional information about the objects on the map. Each database record is associated with the object on the map. This makes it possible to realize the information search function:

1) Searching the map information according to a predetermined attribute;

2) Searching attribute information map object specified in the map image.

Fig. 1 shows an example of the search of the map object of the associated record in the database. 


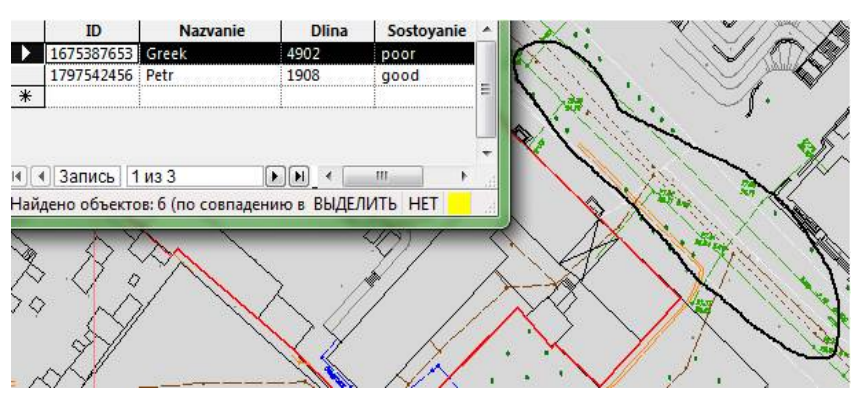

Fig. 1. The link attribute information with the object on the map

Objects of databases it is possible to connect the following methods:

1. symmetrically. For this purpose the fields of records intended for storage of links are added to databases. The code in the field of the link single-digit identifies connection of a graphic primitive with one or several records of the external database;

2. asymmetrically. In this case the link is stored only in one database. Because the link field in other database is absent, referential information includes the list of names of fields and values in this field on which it is possible to find the required records. The mechanism of the asymmetrical binding is built in AutoCad and has the dialogue and program interface. With its help it is possible to connect to primitives of the drawing of record of the external database without modification of its structure.

The external database for any method of binding shall be described as data source. For this purpose use or the DbConnect command, or the built-in editor of files of data sources of Widows OS.

The map base is a plurality of cards upon which the application is achieved. The GIS map may be used in the design of different subject matter, scale. Each card contains a set of layers. For example, GIS «Inventory urban road network based on the GIS technology» contains the following layers :

- The Roadbed;

- Signs;

- Transitions;

- Lights;

- Stop.

Manipulation of the layers make it possible to avoid data redundancy and in minimal times achieve this object.

Thus, the task of design and development of GIS is to solve the individual sub-tasks:

1. Creation of a map basis;

2. Constructioning of the database;

3. Design of the user interface;

4. Development of the functions realized by GIS.

Standard GIS functions (input, deletion, data retrieval, acquisition of look-up information) are implemented similar for different application-oriented tasks. For example, the function of adding the attribute data, the algorithm shown in Fig. 2. Therefore for their development templates are used. It allows to reduce labor input of design. At the same time the specificity of the decision of these tasks is shown in features of data warehousing, visualization of spatial information, analysis methods.

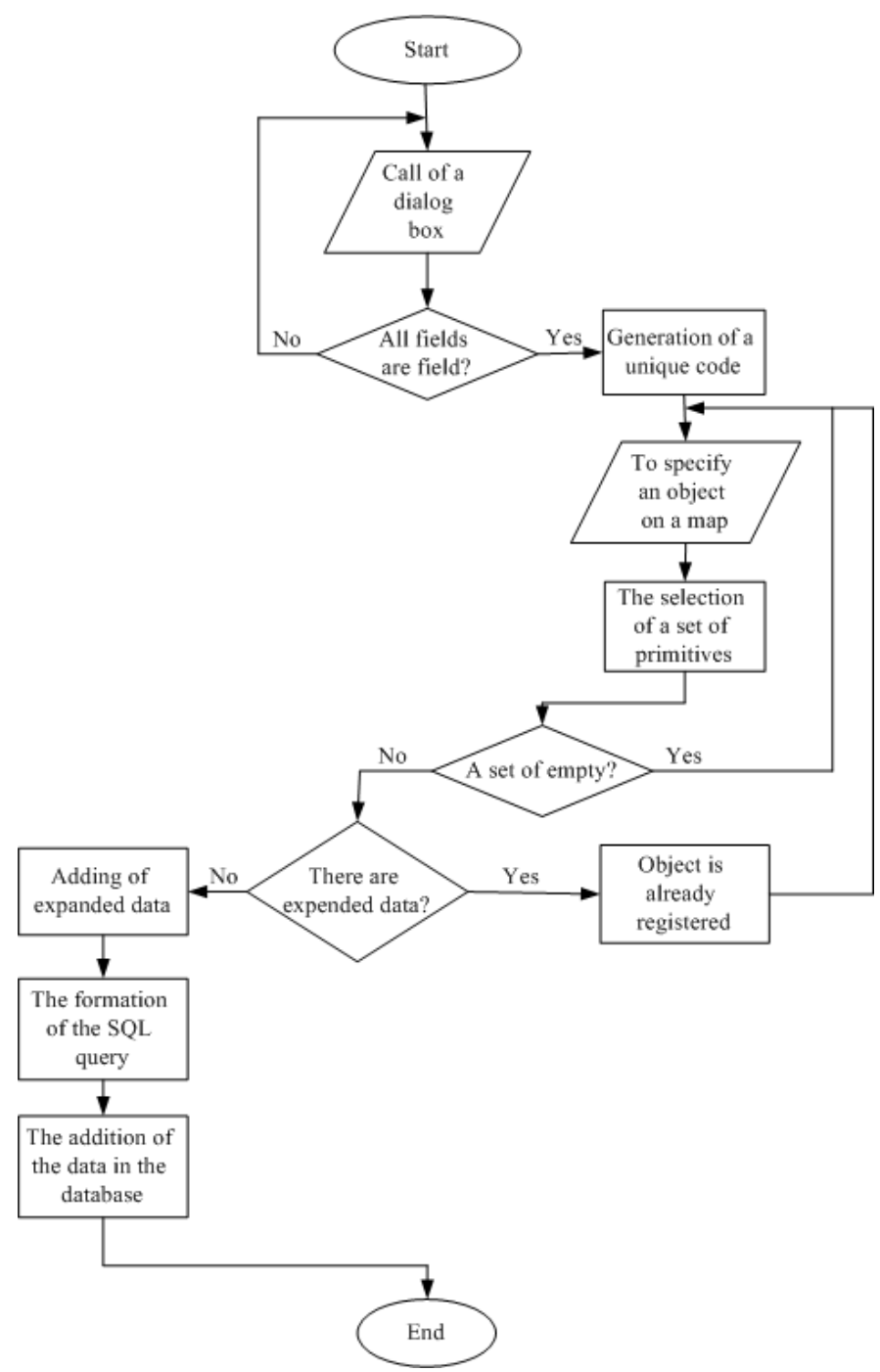

Fig. 2. Algorithm of adding of data

The AutoCad Map 3D is used as a development environment of GIS. It has an open architecture, has a powerful graphics editor and means of three-dimensional simulation. The used built-in AutoLisp programming language relates to languages of artificial simulation and at the same time has means of operation by graphic objects.

Operation with above-mentioned systems is organized on the basis of the following interfaces:

- CAO - the dialogue interface (through DbConnect) access to external databases;

- ASI - the program interface, data access by means of a call of Lisp-functions.

The system works in the multi-user mode. Each type of user operates with GIS via an appropriate interface, which consists of the menu of a call of functions, dialog boxes for outputting the semantic information and the graphical window AutoCad 3D Map. The functional set of GIS also depends on 
type of the user which logs in under the appropriate login and the password.

Distinctive feature of any GIS is integration of cartographical and semantic information. Therefore, step efficient interworking cartographic base with the data store is fundamental in development of GIS.

Fig. 3 is a schematic diagram of an AutoCad Map 3D interaction with the database.

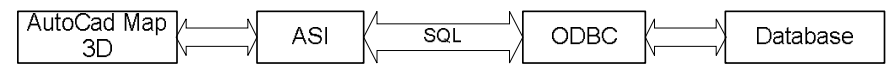

Fig. 3. Structure of interaction of AutoCad Map 3D with the database

In these systems, a database operation is based on the integration of several tools:

- AutoLisp programming Language;

- The set of ODBC drivers (Open Database Connectivity) intended for the organization of the interface mechanism with the DBMS;

- Library of programming of ASI (AutoCad SQL Interface) which allows to provide access to a DB from AutoCad Map 3D. For example, we will give a fragment of a program code for connection establishment with the database:

(arxload "asilisp")

(setq 11 (getvar "acadprefix"))

(setq d2 (substr d1 1 (vl-string-position (ascii ";") d1)))

(setq d3 (substr d2 1 (vl-string-position (ascii " ||$")$ d2 nil T)))

(setq d0 (strcat d3 "||Data Links ||$\left.\left.j e t \_d b s a m p l e s "\right)\right)$

setq $d s$ (asi connect d0)) ;

- SQL query Language.

The relationship between the semantic and Map database is performed based on the extended data of graphic primitives of the environment AutoCad Map 3D.

We will consider a fragment of a program code of adding of expanded data for a cartographical object [2-4]:

(setq lastent (entget (entlast)))

; To get list of attributes of the last entered primitive

(regapp "NEWDATA")

; The application name on which further it is possible to obtain expanded data is registered

$$
\begin{aligned}
& \text { (setq exdata } \\
& ((-3)(" N E W D A T A "(1000 \text {. "Text") } \\
& \text { )) }
\end{aligned}
$$$$
\text { ) }
$$

; The list corresponding to the expanded data including a line is built. The code- 3 corresponds to expanded data, a code 1000 - to string data type.

$$
\begin{aligned}
& \text { (setq newent } \\
& \text { (append lastent exdata)) } \\
& \text { (entmod newent) }
\end{aligned}
$$

; The list of expanded data is added to the attribute list of a primitive and the received list is transferred to the "entmod" function executing modification of the cartographical database.

It is important to mark that each entry in the database is unique. The identification code is assigned for it. Generation of a unique code is realized on the basis of a system date and time:

$$
\text { (setq s (rtos (getvar "Date") 2 15)) }
$$

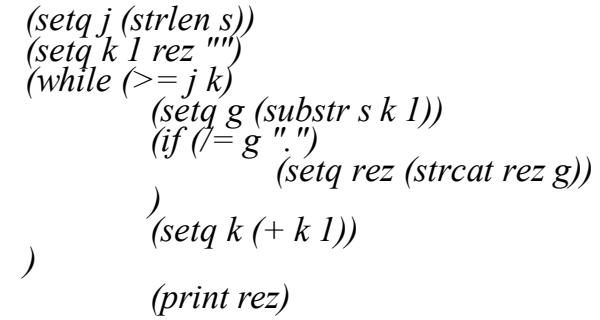

The user operates a GIS via user interface that consists of a call of the menu of functions (fig. 4), dialog boxes intended for data exchange with the user and the graphic AutoCad Map 3D window. It should be noted that the AutoCad Map 3D medium makes it possible to develop the user interface at two levels.

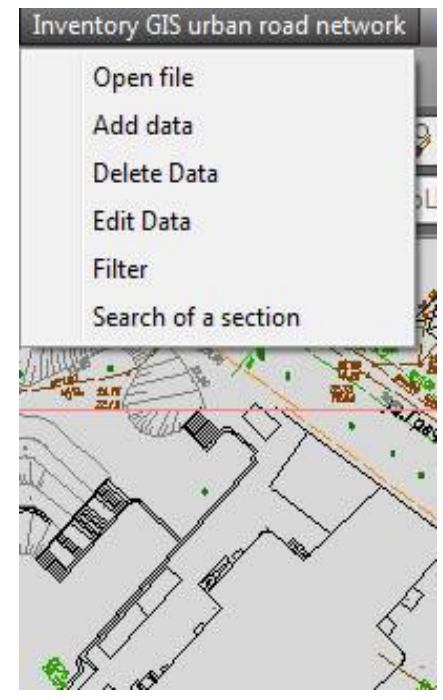

Fig. 4. User menu for GIS « Inventory urban road network»

First, at the level of controls of the main AutoCad window. Menus (context-sensitive and pop-up), a tape, toolbars, sets of the hot keys belong to these elements. These elements include a menu context and pop, a tape, a toolbar, a hotkey sets. The task of designing an interface consists in choosing an appropriate application types of interface elements, placing them in the main window, comparing the desired properties and behavior. This level of development allows to provide the same commands of system differently to different user groups. For example, do not include a command of search and selection of objects in the interface of the operator of data; at the same time for the user having access to these commands of change of information basis of system can be excluded.

Secondly, at the level of individual commands AutoCad Map 3D. During the execution of the instruction some data can be requested from the user. For example, the instruction is a polyline structure PLINE requests coordinates, outputting the request into a command line AutoCad Map 3D. The plotting command outputs a series of Hatch shading windows presenting graphically samples hatching, requesting method indicate the boundaries of the hatching, etc. This level of interface development is directed to constructing a rational user dialog for reliable instruction execution.

Both the level of development of complement each other. The means of combining these levels gives ergonomic and intuitive user interface with the system. 
For creation and editing the user interface in AutoCad Map 3D we use the special editor which is triggered by the CUI command. By means of this editor it is possible to modify any elements of the user interface: menu, toolbars, tape contents, etc.

The description of a dialog is built of the commands in language DCL setting the name of a graphic object, its property and methods. For example, the program code creates a dialog with the field containing the list.

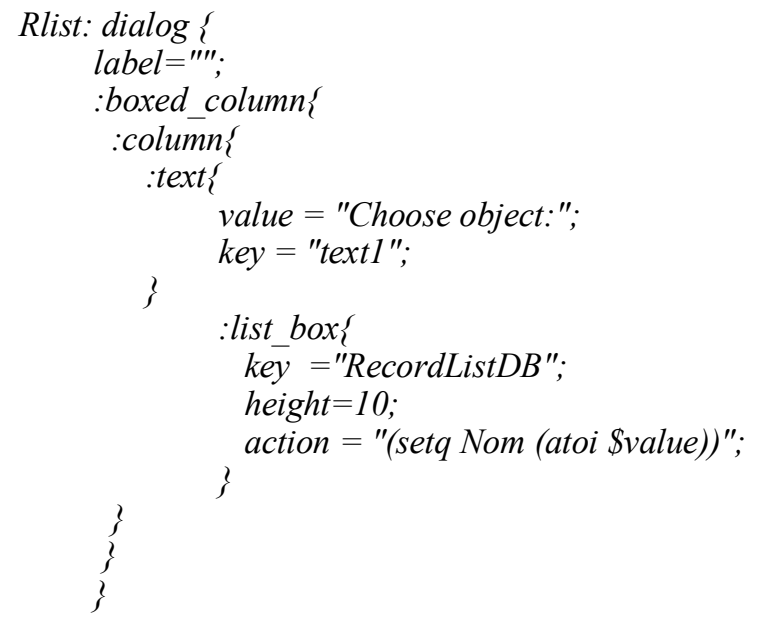

The functional set of the developed GIS includes the fixed functions inherent in all GIS, and the specific functions intended for the decision of the specialized tasks connected to features of application area. Briefly we will describe the functional purpose of the developed elements (modules) of GIS for the decision of specific application-oriented tasks.

The module of GIS «Insurance assessment of vehicles on the basis of GIS-technologies» [5] is intended for decisionmaking by insurance assessment of vehicles. The program allows the determination of the sum of the insurance payments, using data from the database. The program communicates with a structured semantic information in the database with the accounting objects on the map in an AutoCAD and enables visual analysis of spatial objects. The program can be used by insurance company for accounting of the insured vehicles and also for search and selection of information on different criteria, including spatial.

The GIS « Inventory urban road network based on the GIS technology» [6] is for accounting, detection actual state and location of the road sections, road engineering objects belonging to the. Program makes it possible to detect the condition of the road using the geographical data and the database information. The program searches the map objects in Autocad environment over a plurality of predetermined attribute values. The program may be used by governments decision for repairing sections of roads, for automated search and selection of information according to various criteria, including spatial.

The module of GIS «Accounting of objects of the land registry on the basis of geoinformation technologies» is intended for account, detection of actual state and location of objects of the land registry, search and information display about accounting items by different criteria. The program makes it possible to detect the state of the earth areas using data from databases. The program may be used by municipal authorities for control of land fund of municipal unit and also for search and selection of information on different criteria, including spatial.

\section{CONCLUSION}

With the development of these systems have been used as existing templates and development of additional functions for the solution of specific tasks depending on application area was carried out. Application everything modules perhaps by development of intellectual job oriented systems, in particular, in jackets of geographic information systems.

\section{REFERENCES}

[1] Ginis L.A., Gordienko L.V. "Simulation of difficult systems: cognitive set-theoretical approach". Taganrog: Publishing house of the Southern federal university, 2016, $160 \mathrm{p}$.

[2] Poleshchuk N.N., Loskutov P.V. Loskutov. "AutoLisp and VisualLisp in the environment of AutoCad". SPb.: BHV-St. Petersburg, 2006, 960 p.

[3] Zharkov N.V. "AutoCAD 2016. Official Russian version. Effective selfinstruction manual”. SPb.: Science and technology, 2016, 624 p.

[4] Poleshchuk N.N. "Self-instruction manual AutoCAD 2016 (Selfinstruction manual series)". SPb.: BHV-St. Petersburg, 2016, 464 p

[5] Ginis L.A., Gordienko L.V. Certificate on the state registration of the computer program No. 2016616996 of 23.06.2016. "Insurance assessment of vehicles on the basis of GIS-technologies". ROSPATENT, 2016, № 7 (117).

[6] Ginis L.A., Gordienko L.V. Certificate on the state registration of the computer program No. 2016618840 of 09.08 .2016 . "Inventory of a city road network on the basis of GIS-technologies". ROSPATENT, 2016, № 9 (119). 